\title{
Clinical significance of Glasgow Prognostic Score in patients with tuberculous pleurisy
}

\author{
Hye Seon Kang ${ }^{1}$, Hwa Young Lee ${ }^{1}$, Jung Im Jung ${ }^{2}$, Ju Sang Kim ${ }^{1}$, Yong Hyun Kim ${ }^{1}$, Seung Joon Kim ${ }^{1}$, \\ Seok Chan Kim ${ }^{1}$, Soon Seog Kwon ${ }^{1}$, Young Kyoon Kim ${ }^{1}$, Ji Young Kang ${ }^{1}$ \\ ${ }^{1}$ Division of Pulmonary, Allergy and Critical Care Medicine, Department of Internal Medicine, College of Medicine, ${ }^{2}$ Department of Radiology, \\ College of Medicine, The Catholic University of Korea, Seoul, Republic of Korea \\ Contributions: (I) Conception and design: HS Kang, JY Kang; (II) Administrative support: JI Jung, JS Kim, YH Kim; (III) Provision of study \\ materials or patients: SJ Kim, SC Kim, SS Kwon, YK Kim; (IV) Collection and assembly of data: HS Kang, HY Lee, JY Kang; (V) Data analysis and \\ interpretation: JS Kim, YH Kim, JY Kang; (VI) Manuscript writing: All authors; (VII) Final approval of manuscript: All authors. \\ Correspondence to: Ji Young Kang, MD, PhD. Division of Pulmonology, Allergy and Critical Care Medicine, Department of Internal medicine, Seoul \\ St. Mary's Hospital, College of Medicine, The Catholic University of Korea, 222 Banpo-Daero, Seocho-Gu, Seoul 06591, Republic of Korea. \\ Email: rkdwldud@catholic.ac.kr.
}

Background: The Glasgow Prognostic Score (GPS) reflects the host systemic inflammatory response and is a validated, independent prognostic factor for various malignancies. We investigated the clinical significance of the GPS in patients with tuberculosis (TB) pleurisy, focusing on treatment outcomes including paradoxical response (PR).

Methods: This was a retrospective study performed between January 2010 and December 2015 in two referral and university hospitals in South Korea, with intermediate incidences of TB. In all, 462 patients with TB pleurisy were registered in the study. The patients were classified into three groups based on GPS score, as follows: (I) GPS of 2, elevated CRP level $(>1.0 \mathrm{mg} / \mathrm{dL})$ and hypoalbuminemia $(<3.5 \mathrm{~g} / \mathrm{dL})$; (II) GPS of 1, elevated CRP level or hypoalbuminemia; and (III) GPS of 0, neither elevated CRP level nor hypoalbuminemia.

Results: A total of 367 patients with TB pleurisy were finally included. PR occurred in 102 (27.8\%) patients after a mean of 75 days following initiation of anti-TB treatment. The proportion of PR occurrence was significantly lower in the GPS 2 group $(\mathrm{P}=0.007)$. Successful treatment outcomes including cure and completion were also significantly lower in the GPS 2 group $(\mathrm{P}=0.001)$, while all-cause mortality and TB-specific mortality were higher in the GPS 2 group ( $\mathrm{P}=0.001$ and $<0.001$, respectively). Old age over than 65 years old was an independent predicting factor for high mortality and lower PR occurrence. However, the TB relapse rate was not different among the three GPS groups.

Conclusions: Higher GPS value and elderly age were identified as prognostic factors for poor outcomes in TB pleurisy and as predicting factors for lower PR occurrence. More prospective studies are needed to clarify the utility of GPS in patients with TB pleurisy.

Keywords: Albumin; C-reactive protein (CRP); age; tuberculous pleurisy

Submitted Apr 25, 2018. Accepted for publication Sep 27, 2018.

doi: $10.21037 /$ jtd.2018.10.02

View this article at: http://dx.doi.org/10.21037/jtd.2018.10.02

\section{Introduction}

According to the World Health Organization, an estimated 10.4 million new cases of tuberculosis (TB) developed and 1.3 million people died from the disease worldwide in 2016 (1).
Among the types of TB, extrapulmonary TB is difficult to diagnose early due to variable manifestations, and it remains a challenging task to collect the necessary samples in patients. TB pleurisy is the second most common extra- 
pulmonary infection globally and the most common one in South Korea $(2,3)$. In South Korea, 2,237 new tuberculous pleurisy cases were reported in 2017, which accounted for $7.9 \%$ of the 28,161 new TB cases and $38.3 \%$ of the 5,847 extrapulmonary TB cases. The incidence rate of total TB cases was 70.4 per 100,000 populations, a decreasing pattern over the 7 year. The multi-drug resistant TB and extensively-drug resistant TB were reported as 689 and 55 cases, respectively with a corresponding prevalence rate of $2.0 \%$ and $0.2 \%$ in 2017 (4).

Identifying the poor prognostic factors in TB is important, because personalized targeting of care in high-risk groups could improve clinical outcomes. In pulmonary TB (PTB), poor prognostic factors include co-infection with human immunodeficiency virus (HIV), sputum non-conversion at the end of the second month, lower body mass index (BMI), and elderly age (5-10). However, with regard to TB pleurisy, there have been few reports published to date to verify poor prognostic factors such as delayed treatment, pulmonary involvement, or presence of Mycobacterium TB (M. TB) in the effusion (11-13).

With exposure to mycobacterial antigens, the immune system is activated, and the resultant inflammatory process cascades into TB pleurisy $(14,15)$. C-reactive protein (CRP) is an acute-phase protein and indicates systemic inflammatory activity in PTB (16-19). CRP is associated with $M . T B$ loads and host immune response and is decreased by anti-TB treatment (20-22). Also, serum albumin, a negative acute-phase protein, which is used as a marker of inflammation and reflects body mass or malnutrition, is associated with poor outcomes in smearpositive PTB patients $(23,24)$.

The Glasgow Prognostic Score (GPS) is an inflammationbased prognostic scoring system that considers albumin and CRP levels. Recent studies have shown that the GPS represents prognostic factors reliably in patients with various malignancies and acute exacerbation of idiopathic pulmonary fibrosis (25-29). However, to our knowledge, the prognostic value of the GPS in TB pleurisy has never been investigated.

Paradoxical response (PR) is a common phenomenon not only in HIV-positive patients, but also in HIV-negative subjects during TB treatment $(30,31)$. However, despite the good prognosis of PR, physicians may remain challenged because there are some similar situations that can present and confound evaluation, such as combined infection or poor disease control due to noncompliance or drug resistance. Thus, it may be helpful in practice to identify vulnerable patients in whom PR occurs frequently.

In the present study, we evaluated the clinical significance of GPS in patients with TB pleurisy, focusing particularly on treatment outcomes including PR.

\section{Methods}

This was a retrospective study conducted in Seoul St. Mary's Hospital and Bucheon St. Mary's Hospital, which are referral and university hospitals in South Korea, identified as having intermediate incidences of TB between January 2010 and December 2015. The study was approved by the institutional review boards of the participating centers (approval No. XC18RCDI0002). The requirement for informed patient consent was waived by the boards.

\section{Patients}

Patients who were older than 18 years and who had been diagnosed with TB pleurisy were included in our analysis. The serostatus of HIV was tested in all participants. A diagnosis of TB pleurisy was made based on microbiologic evidence of the existence of $M$. TB or positivity for $M$. TB polymerase chain reaction (PCR) in sputum, pleural fluid, or pleural biopsy specimens. Other supporting evidence in diagnosing the disease included the demonstration of TB granulomas with or without caseous necrosis in the pleural tissue or an elevated ADA level that exceeded $40 \mathrm{U} / \mathrm{L}$ with lymphocyte-predominant ( $>50 \%$ of white blood cells in the pleural fluid) exudate in the pleural fluid and clinical improvement after standard anti-TB therapy $(14,32)$. Subjects who demonstrated pulmonary involvement were included, while those who presented with TB empyema with puslike pleural fluid were excluded. Also, those who defaulted during follow-up were not included in the final analysis. The follow-up period ended on December 31, 2017.

\section{Data}

We extracted the following data from patient medical records: patient demographics; smoking and past TB history; comorbid diseases; subjective symptoms; laboratory data (pleural effusion analysis, albumin, and CRP levels in serum); microbiologic assays of TB; drug susceptibility test of $M$. TB; radiologic findings including the sites of pleural effusion and combined PTB; histologic examination in pleural tissue; occurrence of PR; and treatment outcomes.

Acid-fast bacilli (AFB) culture was performed using both 
Ogawa solid media and mycobacteria growth indicator tube (MGIT) liquid media. Drug sensitivity test was performed using the absolute concentration method with the Ogawa media and BACTEC ${ }^{\text {TM }}$ MGIT $^{\text {TM }} 960$ system (Becton Dickinson Sparks, Glencoe, MD, USA) with liquid media. TB PCR was performed using the AdvanSure TB/nontuberculous mycobacterium PCR (LG Life Sciences, Seoul, South Korea) or the Xpert $M$. TB/resistance to rifampin assay (Cepheid, Sunnyvale, CA, USA). PR was defined as a worsening of clinical or radiological findings at two weeks after the initiation of anti-TB treatment in the absence of evidence of disease relapse or the presence of another diagnosis (33). PR was evaluated by two clinicians and one radiologist. The treatment outcomes were categorized into two groups: (I) successful outcomes, such as if TB patients were cured or completed treatment; and (II) unsuccessful outcomes, such as if treatment resulted in treatment failure, default on follow-up, or death. Mortality was classified as TB-specific or non-TB-specific death. Relapse was defined as a TB patient who was declared cured or who had completed treatment as determined by a physician, but who was diagnosed with a recurrent episode of TB during the follow-up period. Following evaluation, the patients were classified into three groups based on GPS score, as follows: (I) GPS of 2, elevated CRP level $(>1.0 \mathrm{mg} / \mathrm{dL})$ and hypoalbuminemia ( $<3.5 \mathrm{~g} / \mathrm{dL})$; (II) GPS of 1 , elevated CRP level or hypoalbuminemia; and (III) GPS of 0 , neither elevated CRP level nor hypoalbuminemia (34,35). The value of serum CRP concentration was measured within 1 week before anti-TB treatment. In our study, CRP was measured by different methods depending on the institutions because two institutions have registered patients. In one institution, high sensitivity CRP (hs-CRP) was measured, while in others, CRP was measured. Hs-CRP levels were measured using a latex immunoassay method with the commercial kits LT CRP-HS II (Wako Pure Chemical Industries, Ltd., Osaka, Japan). Serum CRP levels were measured using immunoturbidimetric assays (CRPL3, Roche, Diagnostics, Indianapolis, IN, USA). In the case of high-sensitivity CRP, upper range of normal value is $0.47 \mathrm{mg} / \mathrm{dL}$, and that for CRP is $5 \mathrm{mg} / \mathrm{L}$. In various clinical conditions, significant correlation was noted between hs-CRP and standard CRP assay after unit conversion (36-38).

\section{Statistical analysis}

Baseline demographics and clinical outcomes were compared among the participants according to GPS criteria. We used
Pearson's Chi-square test to compare discrete variables and Student's $t$-test or analysis of variance to compare continuous variables. Hazard ratios and corresponding $95 \%$ confidence intervals (CIs) were calculated for predictors that were significant in the multivariate analysis. A two-sided $\mathrm{P}$ value $<0.05$ was considered to be statistically significant. All statistical analyses were performed using SPSS for Windows software (ver. 20.0; IBM Corp., Armonk, NY, USA).

\section{Results}

Overall, 462 patients were diagnosed with TB pleurisy, of whom 95 met the exclusion criteria; specifically, these individuals defaulted on follow-up, were transferred to another medical center, or demonstrated a lack of measurements of albumin or CRP level. Thus, 367 patients were finally included in our analysis. The mean age of the included subjects was 40.5 years (range, 19-97 years), and $56.9 \%$ of them were male. Of these patients, $119(32.4 \%)$, $198(54.0 \%)$, and $50(13.6 \%)$ patients were classified into the GPS 0, GPS 1, and GPS 2 groups, respectively. The total follow-up time was 20.6 person-months.

Baseline characteristics and comparisons between the groups are demonstrated in Table 1. Mean age and proportion of diabetes mellitus (DM) were higher in the GPS 2 group $(\mathrm{P}<0.001$ and $\mathrm{P}=0.043$, respectively). The proportions of male gender, smoking history, presence of underlying disease other than DM, history of TB, presenting symptoms, and effusion sites were not different among the three groups (Table 1).

The yields of microbiology and pleural biopsy in the diagnosis of TB pleurisy and laboratory findings according to GPS group are summarized in Table 2. Among the 367 patients with TB pleurisy, 132 (36.0\%) showed culture positivity for $M . T B$ in the effusion. In the 235 patients with negative effusion cultures, 70 had positive sputum cultures. The combined diagnostic yields including AFB culture, smear, and TB PCR were $39.8 \%$ and $38.4 \%$ for the effusion and sputum, respectively. A combination of the effusion and sputum microbiologic results showed a diagnostic sensitivity of $59.1 \%$. The diagnostic yield was $44.4 \%$ (55/124) for histologic confirmation of pleural biopsies. However, these diagnostic results did not differ among the three GPS groups.

In analysis of pleural fluid, the proportion of lymphocytes in inflammatory cells was higher and that of eosinophil was lower in the GPS 2 group compared with the other groups $(\mathrm{P}=0.024$ and 0.005 , respectively). The level of protein was 
Table 1 Baseline characteristics of patients with TB pleurisy

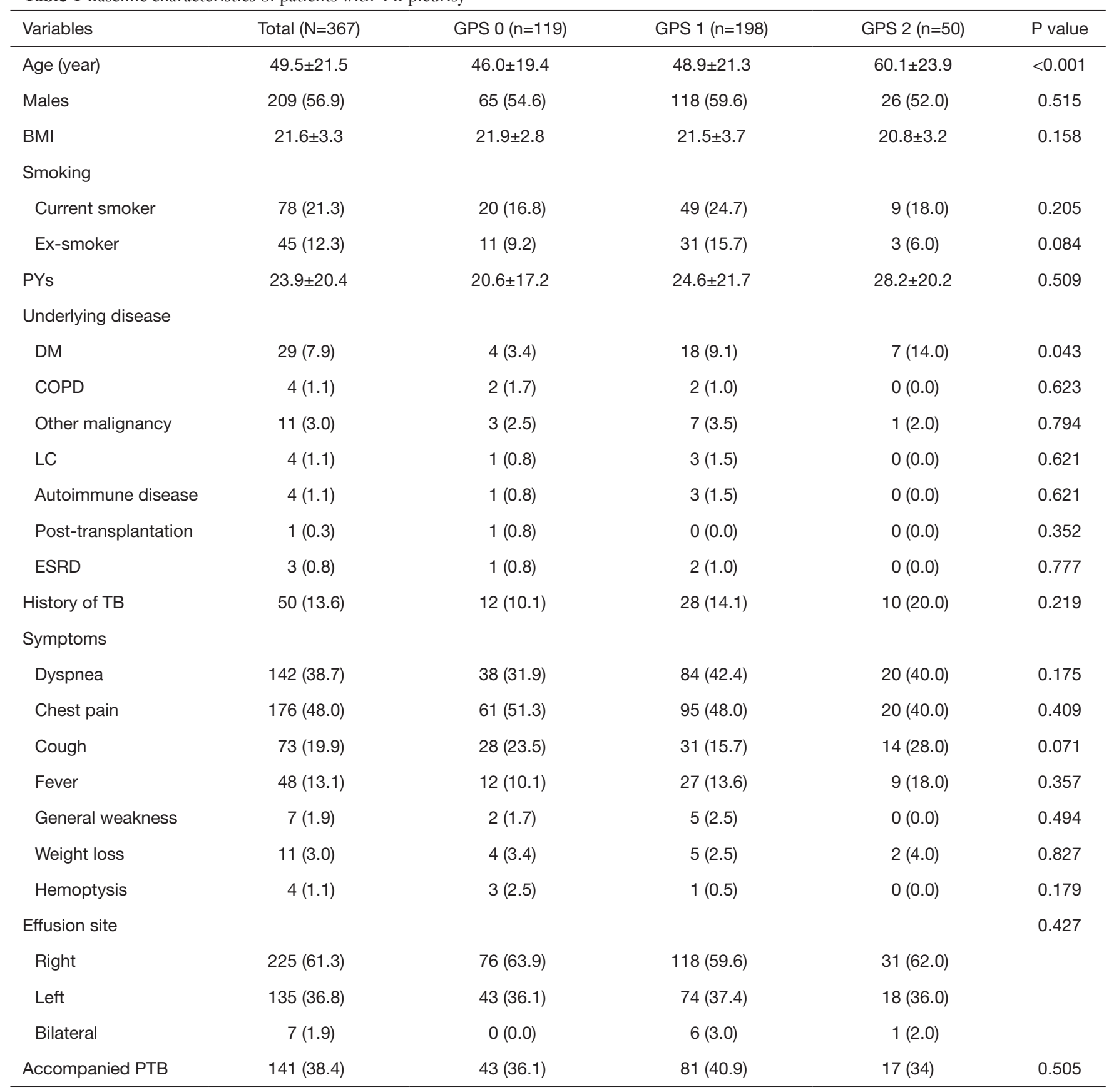

Data are presented as $\mathrm{n}(\%)$ or mean \pm SD. GPS, Glasgow Prognostic Score; BMI, body mass index; PYs, pack years; DM, diabetes mellitus; COPD, chronic obstructive pulmonary disease; LC, liver cirrhosis; ESRD, end stage renal disease; TB, tuberculosis; PTB, pulmonary tuberculosis.

also lower in the GPS 2 group than in the other groups $(\mathrm{P}<0.001)$. Additionally, in serum analysis, the proportion of polymorphonuclear cells among white blood cells was higher and that of lymphocytes was lower in the GPS 2 group $(\mathrm{P}<0.001$ and $<0.001$, respectively). In all three GPS groups, the percentage of lymphocytes in the pleural fluid had an inverse correlation with that in the serum, reflecting a recruitment of inflammatory cells to the TB infection site 
Table 2 Laboratory findings at the time of diagnosis of TB pleurisy according to GPS

\begin{tabular}{|c|c|c|c|c|c|}
\hline Diagnostic procedures & Total $(\mathrm{N}=367)$ & GPS $0(n=119)$ & GPS $1(n=198)$ & GPS $2(n=50)$ & $P$ value \\
\hline Positive effusion $M . T B$ culture & $132(36.0)$ & $47(39.5)$ & $70(35.4)$ & $15(30.0)$ & 0.467 \\
\hline Positive pleural AFB stain & $1(0.3)$ & $0(0.0)$ & $1(0.5)$ & $0(0.0)$ & 0.651 \\
\hline Pleural TB PCR & $34(9.3)$ & $4(3.4)$ & $26(13.1)$ & $4(8.0)$ & 0.014 \\
\hline Negative effusion $M$. TB culture & 232 (63.2) & $71(59.7)$ & $126(63.6)$ & $35(70.0)$ & 0.467 \\
\hline Positive sputum AFB culture & 70 & $18 / 71(25.4)$ & $42 / 126(33.3)$ & 10/35 (28.6) & 0.459 \\
\hline Sputum AFB culture & $128(34.9)$ & $41(34.5)$ & $72(36.4)$ & $15(30.0)$ & 0.663 \\
\hline Sputum AFB stain & $2(0.5)$ & $1(0.8)$ & $1(0.5)$ & $0(0.0)$ & 0.792 \\
\hline $\begin{array}{l}\text { Combination of effusion and sputum } \\
\text { AFB smear/culture/TB PCR }\end{array}$ & $217(59.1)$ & $66(55.5)$ & $124(62.6)$ & $27(54.0)$ & 0.275 \\
\hline \multicolumn{6}{|l|}{ Pleural fluid analysis } \\
\hline $\mathrm{pH}$ & & $7.5 \pm 0.3$ & $7.7 \pm 0.5$ & $7.5 \pm 0.4$ & $<0.001$ \\
\hline WBC $\left(\times 10^{3}\right)$ & & $3,304.9 \pm 2,963.1$ & $3,188.7 \pm 4,156.2$ & $2,561.6 \pm 3,056.4$ & 0.47 \\
\hline Segment neutrophil (\%) & & $10.4 \pm 12.1$ & $12.7 \pm 18.6$ & $9.4 \pm 17.1$ & 0.32 \\
\hline Lymphocyte (\%) & & $73.3 \pm 19.3$ & $78.1 \pm 21.3$ & $82.2 \pm 18.9$ & 0.024 \\
\hline Eosinophil (\%) & & $2.4 \pm 7.8$ & $0.5 \pm 3.6$ & $0.1 \pm 0.3$ & 0.005 \\
\hline WBC $\left(\times 10^{3}\right)$ & & $6,498.8 \pm 1,684.1$ & $6,765.4 \pm 2,040.1$ & $7,277.0 \pm 1,843.1$ & 0.053 \\
\hline Segment neutrophil (\%) & & $64.0 \pm 8.8$ & $67.2 \pm 9.1$ & $70.0 \pm 10.3$ & $<0.001$ \\
\hline Lymphocyte (\%) & & $22.1 \pm 7.5$ & $18.7 \pm 7.5$ & $15.9 \pm 7.1$ & $<0.001$ \\
\hline
\end{tabular}

Data are presented as $n$ (\%) or mean \pm SD. TB, tuberculosis; GPS, Glasgow Prognostic Score; M. TB, Mycobacterium tuberculosis; AFB, Acid-Fast Bacilli; PCR, polymerase chain reaction; pH, potential of hydrogen; WBC, white blood cells; LDH, lactate dehydrogenase; ADA, adenosine deaminase.

from the systemic circulation (Table 2).

PR occurred in $102(27.8 \%)$ patients after a mean of 75 days following initiation of anti-TB treatment. The proportion of $\mathrm{PR}$ occurrence was significantly lower in the GPS 2 group $(\mathrm{P}=0.007)$. In the patients with $\mathrm{PR}$, $27(26.5 \%)$ demonstrated accompanying complications such as peripleural abscess formation, though surgical intervention was required in only four patients. We used the peripleural abscess as empyema encircling the pleura. Drug susceptibility testing was performed on 178 of 213 (83.6\%) cultures positive for $M$. TB, and the results of this testing were as follows: 163 demonstrated all-drugs sensitivity, 8 demonstrated resistance to isoniazid, 2 demonstrated resistance to rifampin, 1 demonstrated resistance to 
Table 3 Comparison of treatment outcomes according to GPS

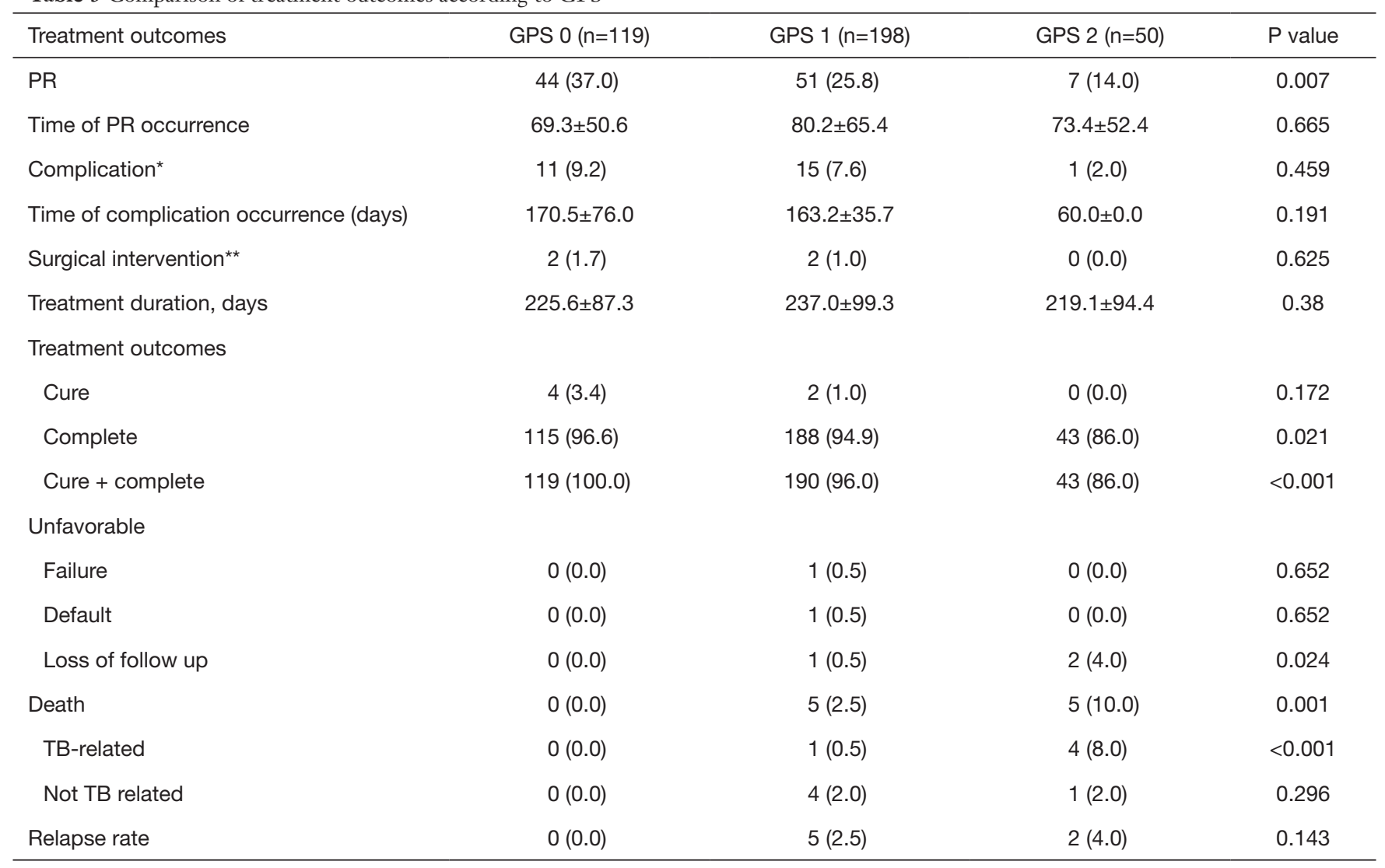

Data are presented as $\mathrm{n}(\%)$ or mean $\pm \mathrm{SD}$. *, complications are peripleural abscess or lung infiltrations; **, surgical intervention includes drainage or decortication. GPS, Glasgow Prognostic Score; PR, paradoxical response; TB, tuberculosis.

pyrazinamide, 3 demonstrated multi-drug-resistant TB, and 1 demonstrated pre-extensively drug-resistant TB. The proportions of drug-resistant TB were not different depending on GPS group. The mean treatment duration was 7.7 months. Out of the 367 patients, 352 (95.9\%) showed successful treatment outcomes, 10 (2.7\%) died during treatment, and 7 (1.9\%) had TB recurrence during the follow-up period. Successful treatment outcome was significantly lower in the GPS 2 group $(\mathrm{P}<0.001)$, and allcause mortality and TB-specific mortality were higher in the GPS 2 group $(\mathrm{P}=0.001$ and $<0.001$, respectively). However, the TB relapse rate was not different among the three groups (Table 3).

In univariate analysis, elderly age $(\mathrm{P}=0.002), \mathrm{DM}$ $(\mathrm{P}=0.019)$, and higher GPS value $(\mathrm{P}=0.008)$ were negative predicting factors for $\mathrm{PR}$ occurrence. In the multivariate analysis, elderly age [odds ratio (OR): $0.469,95 \%$ CI: $0.259-0.850 ; \mathrm{P}=0.013]$ and GPS of 2 (OR: $0.345,95 \%$ CI: $0.140-0.847 ; \mathrm{P}=0.020)$ were independent negative predicting factors for PR occurrence (Table 4). For all-cause mortality, elderly age $(\mathrm{P}=0.003)$ and GPS of $2(\mathrm{P}=0.003)$ were prognostic factors for poor clinical outcomes in univariate analysis. In multivariate analysis, elderly age (OR: 20.328, 95\% CI: 2.507-164.833, $\mathrm{P}=0.005$ ) and GPS of 2 (OR: 4.813 , 95\% CI: $1.272-18.207, \mathrm{P}=0.021$ ) were also independent prognostic factors for all-cause mortality in TB pleurisy (Table 5).

\section{Discussion}

In the present study, we compared the clinical characteristics and treatment outcomes of TB pleurisy with respect to GPS value. According to our findings, the proportions of patients in the GPS 0, GPS 1, and GPS 2 groups were 119 (32.4\%), 198 (54.0\%), and 50 (13.6\%), respectively. Regarding the total study cohort, PR and death occurred in $102(27.8 \%)$ cases and $10(2.7 \%)$ cases, respectively, with patients with GPS 2 having significantly lower PR occurrence (14.0\%) and 
Table 4 Univariate and multivariate analysis of predicting factors for paradoxical response in patients with TB pleurisy

\begin{tabular}{|c|c|c|c|c|c|c|}
\hline Factor & \multicolumn{3}{|c|}{ Univariate analysis } & \multicolumn{3}{|c|}{ Multivariate analysis } \\
\hline Older age (>65 years) & 0.398 & $0.223-0.711$ & 0.002 & 0.469 & $0.259-0.850$ & 0.013 \\
\hline DM & 0.175 & $0.041-0.749$ & 0.019 & 0.232 & $0.053-1.013$ & 0.052 \\
\hline \multicolumn{7}{|l|}{ GPS } \\
\hline 1 & 0.600 & $0.367-0.979$ & 0.041 & 0.669 & $0.406-1.104$ & 0.116 \\
\hline 2 & 0.277 & $0.115-0.670$ & 0.004 & 0.345 & $0.140-0.847$ & 0.020 \\
\hline Positive pleural M. TB culture & 1.016 & $0.631-1.637$ & 0.947 & - & - & - \\
\hline
\end{tabular}

OR, odds ratio; Cl, confidence interval; DM, diabetes mellitus; GPS, Glasgow Prognostic Score; M. TB, Mycobacterium tuberculosis.

Table 5 Univariate and multivariate analysis of risk factors for all-cause mortality in patients with TB pleurisy

\begin{tabular}{|c|c|c|c|c|c|c|}
\hline Factor & \multicolumn{3}{|c|}{ Univariate analysis } & \multicolumn{3}{|c|}{ Multivariate analysis } \\
\hline Older age (>65 years) & 24.469 & $3.059-195.698$ & 0.003 & 20.328 & $2.507-164.833$ & 0.005 \\
\hline DM & 1.306 & $0.160-10.680$ & 0.803 & - & - & - \\
\hline \multicolumn{7}{|l|}{ GPS } \\
\hline 2 & 6.933 & $1.931-24.896$ & 0.003 & 4.813 & $1.272-18.207$ & 0.021 \\
\hline Positive M. TB pleural culture & 1.419 & $0.374-5.378$ & 0.607 & - & - & - \\
\hline
\end{tabular}

OR, odds ratio; CI, confidence interval; DM, diabetes mellitus; GPS, Glasgow Prognostic Score; M. TB, Mycobacterium tuberculosis.

higher mortality rates (10\%) than those with GPSs of 0 or 1.

One possible explanation for the association between mortality and higher GPS value is that host systemic inflammation and mycobacterial control are strongly related in patients with TB pleurisy. In previous clinical studies, CRP level was found to be significantly decreased after initiation of anti-TB therapy (22). High CRP level is associated with mycobacterial load, and sustained elevated level of CRP is related to delayed culture conversion after treatment. It has been suggested that $M$. TB-driven inflammatory tissue damage may trigger induction of CRP production (18). Lower levels of serum albumin have also been strongly associated with mortality in patients with severe TB $(24,39)$. Tsukahara et al. reported that a prognostic nutritional index consisting of albumin and lymphocyte count is a relating factor for poor prognosis in smear-positive PTB (23). Taken together, the results indicate that, as the GPS consists of CRP level and albumin, higher GPS values are associated with increased mortality.

In addition, the mean age was higher in the GPS 2 group. $\mathrm{TB}$ in the elderly is related to atypical clinical and radiologic features, so it is often associated with a delay in diagnosis, resulting in higher morbidity and mortality $(40,41)$. Hypoalbuminemia is known to be more prevalent in elderly individuals who are frail compared with those who are healthy. In healthy elderly males, high CRP level and hypoalbuminemia increased the risk of early death (42). A higher GPS value was associated with elderly age in our study; however, elderly age and higher GPS values were independent predicting factors for mortality in patients with TB pleurisy.

Even though the exact mechanism remains uncertain, $\mathrm{PR}$ is known to be related to an enhanced immunological response to large amounts of tuberculoprotein release prompted by effective anti-TB treatment (43). HIV coinfection is a well-recognized risk factor for PR, usually 
developed when concomitant anti-retroviral therapy is initiated. Some studies have reported that there are various risk factors that predict $\mathrm{PR}$ in patients with $\mathrm{TB}$, including young age, positive TB culture, presence of lymph node disease, hypo/hyperalbuminemia, and lymphopenia $(30,44-47)$. Also, anemia and the proportions of eosinophils and protein concentration in pleural fluid at the time of diagnosis is known to be associated with PR $(30,45)$. Some researchers observed that, in HIV-coinfected TB patients, higher CRP level was related to the risk of developing PR $(48,49)$. In our study, rather high number of PR was presented. In South Korea, two studies reported PR was developed with an incidence of $23 \%$ and $16 \%$ in HIV-negative patients with TB pleurisy, respectively. Our incidence of PR was higher than those with the past studies. In fact, most clinicians in our institutions followed up a chest X-ray at the initiation, 2 weeks or 1 months later, 2 months later of the anti-TB treatment during the intensive phase, with laboratory test including sputum studies, and then at monthly or bimonthly during the continuation phase until the treatment completion. This strategy to monitor TB patients might detect frequently asymptomatic or mild PR cases. We found that the GPS 2 group in our study demonstrated a lower PR occurrence compared with the GPS 0 and 1 groups. There are several explanations for these results. First, serum albumin is not simply a negative acute-phase protein, but also represents nutrition status. In an experimental TB model, the immune system in patients with malnutrition may lose control over $M$. $T B$ because of the suppression of T-cells caused by decreasing lymphocyte stimulation, presence of $\mathrm{T}$ helper 1 cytokines, and elevated production of transforming growth factor- $\beta$ (50). Therefore, in the present study, the presence of hypoalbuminemia, which indicates a decreased nutritional and immunologic status, may play a part in the lower occurrence of PR, reflecting the severity of systemic inflammation in the GPS 2 group. Second, the GPS 2 group contained a considerably higher number of elderly people than did the other two groups. In our study, elderly age was one of the factors associated with mortality and lower PR occurrence. In previous investigations, younger age was found to be predictive for PR $(47,51)$. Aging is related to a decline in host defense mechanisms and altered immune function (52). Low PR occurrence in the elderly is possibly related to decreased immunologic status. However, to verify a possible relationship between the GPS and PR occurrence, further prospective studies are necessary.

In the present study, we confirmed TB pleurisy in $59.5 \%(217 / 367)$ of participants according to the obtained microbiologic evidence. On the other hand, the percentage of cases diagnosed by only a compatible pathology via pleural biopsy was $44.3 \%$ (55/124). This high performance in diagnosing TB pleurisy by microbiologic results could be due to the introduction of an automated liquid culture system for M. TB and advanced TB PCR tests (13). These findings provide evidence that recent microbiologic study is comparably useful-as much so as an invasive histologic examination-in the diagnosis of TB pleurisy. In line with the higher detection of $M . T B$ in pleural fluid than previously reported, Ruan et al. suggested that TB pleurisy is not a delayed hypersensitivity reaction to $\mathrm{TB}$ antigens, but rather a continuous spectrum of the disease that is initiated by a direct infection of $M . T B$ in the pleural space and that evolves with a cascade of immunological responses (13). They also reported that patients with $M . T B$ in the pleural fluid had a higher mortality rate than did those without it. However, we did not identify any association between positive culture for $M . T B$ and death in the present study (data not shown). Our data imply that the more significant item to consider in the clinical outcome of TB pleurisy might be the host's inflammatory response to the pathogen rather than the existence of $M . T B$.

Our study had certain limitations. First, it is a retrospective study with inherent weakness. Nonetheless, it is a multicenter study that included a relatively large sample size and that provides valuable clinical information about the role of GPS in TB pleurisy. Second, GPS monitoring was not performed serially according to the time of $\mathrm{PR}$ occurrence and mortality. As we mentioned above, the consideration of inflammatory markers might be useful in evaluating treatment response in TB patients. Third, the association of GPS with mortality in TB pleurisy patients could be explained by unassessed confounding factors.

Despite these limitations, our study had a number of strengths. Although several studies exploring prognostic factors in patients with TB pleurisy have been performed, the prognostic value of GPS has not been previously investigated. Our study suggests that higher GPS value in the pretreatment phase is a poor prognostic factor for clinical outcomes and is related with lower PR occurrence. The GPS can be determined through a simple, easy, and inexpensive test and is a reliable marker of inflammatory activity. Therefore, the GPS could help clinicians in predicting the clinical course of patients with TB pleurisy 
and assist them to more successfully manage high-risk populations.

In conclusion, higher GPS value and elderly age were identified as prognostic factors for poor outcomes in TB pleurisy and as predicting factors for lower PR occurrence. Additional prospective studies are needed to clarify the utility of GPS in patients with TB pleurisy.

\section{Acknowledgements}

Funding: This research was supported by a grant of the Korea Health Technology R\&D Project through the Korea Health Industry Development Institute (KHIDI), funded by the Ministry of Health \& Welfare, Republic of Korea (grant number: HI14C1234).

\section{Footnote}

Conflicts of Interest: The authors have no conflicts of interest to declare.

Ethical Statement: The study was approved by the institutional review boards of the participating centers (approval No. XC18RCDI0002). The requirement for informed patient consent was waived by the boards.

\section{References}

1. World Health Organization. Global tuberculosis report 2017. In: WHO 2017. Available online: http://www. who.int/tb/publications/global_report/en/. Accessed 5 February 2018.

2. Sharma SK, Mohan A. Extrapulmonary tuberculosis. Indian J Med Res 2004;120:316-53.

3. Korea Centers for Disease Control and Prevention. Annual report on the notified tuberculosis in Korea 2016. 2017. Available online: http://cdc.go.kr/CDC/ info/CdcKrInfo0302.jsp? menuIds=HOME001MNU1132-MNU1138-MNU0038\&fid=32\&q_ type $=\&$ q_value $=\& \operatorname{cid}=73878 \&$ pageNum $=$. Accessed 5 February 2018.

4. Korean Centers for Disease Control and Prevention. Notified tuberculosis status in Korea. Centers for Disease Control and Prevention, Cheongju. 2018. Available online: http://tbzero.cdc.go.kr/tbzero/board/boardView.do

5. Mbatchou Ngahane BH, Dahirou F, Tchieche C, et al. Clinical characteristics and outcomes of tuberculosis in Douala, Cameroon: a 7-year retrospective cohort study.
Int J Tuberc Lung Dis 2016;20:1609-14.

6. Adamu AL, Gadanya MA, Abubakar IS, et al. High mortality among tuberculosis patients on treatment in Nigeria: a retrospective cohort study. BMC Infect Dis 2017;17:170.

7. Heunis JC, Kigozi NG, Chikobvu P, et al. Risk factors for mortality in TB patients: a 10-year electronic record review in a South African province. BMC Public Health 2017;17:38.

8. Lai HH, Lai YJ, Yen YF. Association of Body Mass Index with Timing of Death during Tuberculosis Treatment. PLoS One 2017;12:e0170104.

9. Takarinda KC, Sandy C, Masuka N, et al. Factors Associated with Mortality among Patients on TB Treatment in the Southern Region of Zimbabwe, 2013. Tuberc Res Treat 2017;2017:6232071.

10. Yen YF, Feng JY, Pan SW, et al. Determinants of mortality in elderly patients with tuberculosis: a population-based follow-up study. Epidemiol Infect 2017;145:1374-81.

11. Lin MT, Wang JY, Yu CJ, et al. Mycobacterium tuberculosis and polymorphonuclear pleural effusion: incidence and clinical pointers. Respir Med 2009; 103:820-6.

12. Shu CC, Wang JT, Wang JY, et al. In-hospital outcome of patients with culture-confirmed tuberculous pleurisy: clinical impact of pulmonary involvement. BMC Infect Dis 2011;11:46.

13. Ruan SY, Chuang YC, Wang JY, et al. Revisiting tuberculous pleurisy: pleural fluid characteristics and diagnostic yield of mycobacterial culture in an endemic area. Thorax 2012;67:822-7.

14. Gopi A, Madhavan SM, Sharma SK, et al. Diagnosis and treatment of tuberculous pleural effusion in 2006. Chest 2007;131:880-9.

15. Shu CC, Wang JY, Hsu CL, et al. Diagnostic role of inflammatory and anti-inflammatory cytokines and effector molecules of cytotoxic $\mathrm{T}$ lymphocytes in tuberculous pleural effusion. Respirology 2015;20:147-54.

16. Yoon C, Semitala FC, Atuhumuza E, et al. Point-of-care C-reactive protein-based tuberculosis screening for people living with HIV: a diagnostic accuracy study. Lancet Infect Dis 2017;17:1285-92.

17. Yoon C, Chaisson LH, Patel SM, et al. Diagnostic accuracy of C-reactive protein for active pulmonary tuberculosis: a meta-analysis. Int J Tuberc Lung Dis 2017;21:1013-9.

18. Miranda P, Gil-Santana L, Oliveira MG, et al. Sustained elevated levels of $\mathrm{C}$-reactive protein and ferritin in pulmonary tuberculosis patients remaining culture positive 
upon treatment initiation. PLoS One 2017;12:e0175278.

19. Lee J, Yoo SS, Lee SY, et al. Pleural fluid adenosine deaminase/serum C-reactive protein ratio for the differentiation of tuberculous and parapneumonic effusions with neutrophilic predominance and high adenosine deaminase levels. Infection 2017;45:59-65.

20. Almani SA, Shaikh TZ, Khoharo HK, et al. Serum enolase-2, high-sensitivity C-reactive protein, and serum cholesterol in smear-positive drug-naive pulmonary tuberculosis. J Res Med Sci 2017;22:49.

21. Mesquita ED, Gil-Santana L, Ramalho D, et al. Associations between systemic inflammation, mycobacterial loads in sputum and radiological improvement after treatment initiation in pulmonary TB patients from Brazil: a prospective cohort study. BMC Infect Dis 2016;16:368.

22. Mendy J, Togun T, Owolabi O, et al. C-reactive protein, Neopterin and Beta2 microglobulin levels pre and post TB treatment in The Gambia. BMC Infect Dis 2016;16:115.

23. Tsukahara T, Horita N, Tashiro K, et al. Factors for Predicting Outcomes among Non-HIV Patients with Pulmonary Tuberculosis. Intern Med 2017;56:3277-82.

24. Loh WJ, Yu Y, Loo CM, et al. Factors associated with mortality among patients with active pulmonary tuberculosis requiring intensive care. Singapore Med J 2017;58:656-9.

25. Forrest LM, McMillan DC, McArdle CS, et al. Evaluation of cumulative prognostic scores based on the systemic inflammatory response in patients with inoperable nonsmall-cell lung cancer. Br J Cancer 2003;89:1028-30.

26. Machida Y, Sagawa M, Tanaka M, et al. Postoperative survival According to the Glasgow Prognostic Score in Patients with Resected Lung Adenocarcinoma. Asian Pac J Cancer Prev 2016;17:4677-80.

27. Li XH, Chang H, Xu BQ, et al. An inflammatory biomarker-based nomogram to predict prognosis of patients with nasopharyngeal carcinoma: an analysis of a prospective study. Cancer Med 2017;6:310-9.

28. Lindenmann J, Fink-Neuboeck N, Avian A, et al. Preoperative Glasgow Prognostic Score as additional independent prognostic parameter for patients with esophageal cancer after curative esophagectomy. Eur J Surg Oncol 2017;43:445-53.

29. Kang HS, Cho KW, Kwon SS, et al. Prognostic significance of Glasgow prognostic score in patients with acute exacerbation of idiopathic pulmonary fibrosis. Respirology 2018;23:206-12.

30. Jeon K, Choi WI, An JS, et al. Paradoxical response in HIV-negative patients with pleural tuberculosis: a retrospective multicentre study. Int J Tuberc Lung Dis 2012;16:846-51.

31. Al-Majed SA. Study of paradoxical response to chemotherapy in tuberculous pleural effusion. Respir Med 1996;90:211-4.

32. Light RW. Pleural effusions. Med Clin North Am 2011;95:1055-70.

33. Breen RA, Smith CJ, Bettinson H, et al. Paradoxical reactions during tuberculosis treatment in patients with and without HIV co-infection. Thorax 2004;59:704-7.

34. McMillan DC, Crozier JE, Canna K, et al. Evaluation of an inflammation-based prognostic score (GPS) in patients undergoing resection for colon and rectal cancer. Int J Colorectal Dis 2007;22:881-6.

35. McMillan DC. The systemic inflammation-based Glasgow Prognostic Score: a decade of experience in patients with cancer. Cancer Treat Rev 2013;39:534-40.

36. Monneret D, Mestari F, Djiavoudine S, et al. Wide-range CRP versus high-sensitivity CRP on Roche analyzers: focus on low-grade inflammation ranges and highsensitivity cardiac troponin T levels. Scand J Clin Lab Invest 2018;78:346-51.

37. Milone MT, Kamath AF, Israelite CL. Converting between high- and low-sensitivity C-reactive protein in the assessment of periprosthetic joint infection. J Arthroplasty 2014;29:685-9.

38. Helal I, Zerelli L, Krid M, et al. Comparison of C-reactive protein and high-sensitivity C-reactive protein levels in patients on hemodialysis. Saudi J Kidney Dis Transpl 2012;23:477-83.

39. Kang JY, Kim MS, Kim JS, et al. Clinical Characteristics and Prognostic Factors in Patients with Pulmonary Tuberculosis Admitted to Intensive Care Units. Tuberc Respir Dis (Seoul) 2010:259-66.

40. Enarson DA, Grzybowski S, Dorken E. Failure of diagnosis as a factor in tuberculosis mortality. Can Med Assoc J 1978;118:1520-2.

41. Katz I, Rosenthal T, Michaeli D. Undiagnosed tuberculosis in hospitalized patients. Chest 1985;87:770-4.

42. Edvardsson M, Sund-Levander M, Milberg A, et al. Differences in levels of albumin, ALT, AST, gamma-GT and creatinine in frail, moderately healthy and healthy elderly individuals. Clin Chem Lab Med 2018;56:471-8.

43. Campbell IA, Dyson AJ. Lymph node tuberculosis: a comparison of various methods of treatment. Tubercle 1977;58:171-9.

44. Brown CS, Smith CJ, Breen RA, et al. Determinants of treatment-related paradoxical reactions during anti- 
tuberculosis therapy: a case control study. BMC Infect Dis 2016;16:479.

45. Cheng SL, Wang HC, Yang PC. Paradoxical response during anti-tuberculosis treatment in HIV-negative patients with pulmonary tuberculosis. Int J Tuberc Lung Dis 2007;11:1290-5.

46. Cheng VC, Yam WC, Woo PC, et al. Risk factors for development of paradoxical response during antituberculosis therapy in $\mathrm{HIV}$-negative patients. Eur J Clin Microbiol Infect Dis 2003;22:597-602.

47. Jung JW, Shin JW, Kim JY, et al. Risk factors for development of paradoxical response during antituberculosis treatment in HIV-negative patients with pleural tuberculosis. Tohoku J Exp Med 2011;223:199-204.

48. Haddow LJ, Dibben O, Moosa MY, et al. Circulating inflammatory biomarkers can predict and characterize tuberculosis-associated immune reconstitution inflammatory syndrome. Aids 2011;25:1163-74.

49. Narendran G, Andrade BB, Porter BO, et al. Paradoxical tuberculosis immune reconstitution inflammatory syndrome (TB-IRIS) in HIV patients with culture confirmed pulmonary tuberculosis in India and the potential role of IL- 6 in prediction. PLoS One 2013;8:e63541.

50. Schaible UE, Kaufmann SH. Malnutrition and infection: complex mechanisms and global impacts. PLoS Med 2007;4:e115.

51. Cho OH, Park KH, Kim T, et al. Paradoxical responses in non-HIV-infected patients with peripheral lymph node tuberculosis. J Infect 2009;59:56-61.

52. Ben-Yehuda A, Weksler ME. Host resistance and the immune system. Clin Geriatr Med 1992;8:701-11.
Cite this article as: Kang HS, Lee HY, Jung JI, Kim JS, Kim YH, Kim SJ, Kim SC, Kwon SS, Kim YK, Kang JY. Clinical significance of Glasgow Prognostic Score in patients with tuberculous pleurisy. J Thorac Dis 2018;10(11):6077-6087. doi: 10.21037/jtd.2018.10.02 